\title{
Preparation of $\beta$-chitin nanofiber aerogels by lyophilization
}

Shin Suenaga, Mitsumasa Osada*

Department of Chemistry and Materials, Faculty of Textile Science and Technology, Shinshu

University, 3-15-1, Tokida, Ueda, Nagano 386-8567, Japan

Corresponding author. Tel.: +81-268-21-5458; Fax: +81-268-21-5391

E-mail: osadam@shinshu-u.ac.jp 


\section{Highlights}

- $\quad \beta$-Chitin nanofibers differing in $\mathrm{pH}$ values were lyophilized to produce aerogels.

- Effects of the freezing speed and surface charge of chitin nanofiber were studied.

- Morphological transformation occurred in neutral $\beta$-chitin nanofiber aerogels.

- Acidic aerogels possessed uniform microstructure due to electrostatic repulsion.

- Same acidic aerogel also resulted in high mechanical strength and surface area. 


\section{Abstract}

In this study, chitin nanofiber dispersions prepared in neutral and acidic $\mathrm{pH}$ conditions were lyophilized to produce aerogels. The effects of the freezing speed of the nanofiber dispersions and the dispersibility of the chitin nanofiber were studied. The characteristics of the aerogels were studied using scanning electron microscopy, relative surface area measurements, and compression tests. The repulsion forces of the chitin nanofiber in acidic conditions were effective in the formation of a more uniform microstructure during water solidification, resulting in aerogels with a high mechanical strength. The aerogel made from the chitin nanofiber dispersion prepared in neutral conditions was influenced by ice crystal growth during freezing, resulting in a nonuniform structure. In contrast, the surface area of the aerogel in neutral conditions interestingly remained unchanged compared to that of the original powder, which was due to the morphological transformation.

Key words: chitin nanofiber, aerogel, lyophilization 


\section{Introduction}

Aerogels are materials with controllable micro- or nano-sized pores and a high porosity, large specific surface area, and low density [1]. Two major approaches are used to prepare aerogels: one is supercritical drying with carbon dioxide [2], and the other is lyophilization [3]. Supercritical drying can preserve the nanostructure inside the starting material, resulting in a high relative surface area $\left(>300 \mathrm{~m}^{2} \mathrm{~g}^{-1}\right)$ and nanosized pores $(<50 \mathrm{~nm})$ [4,5]. Aerogels made by supercritical drying should be used as sound or heat insulators because of their structural features. In contrast, aerogels made by lyophilization exhibit pores $(10 \mathrm{~nm}-100 \mu \mathrm{m})$ larger than those of the aerogels made by supercritical drying [6,7]. In this case, the pore size and shape depend on the ice crystal growth during freezing and can be controlled by the freezing temperature and speed $[8,9]$. In addition, a xylem-like monolith structure has been reported to be prepared by a unidirectional freeze-drying method [10]. This type of aerogel has been studied for medical applications because of the solute permeability, cell adhesive property, and control of the cell spheroid because the pore size is relatively large, and the pore shape can be controlled.

Aerogels can be produced from inorganic and organic compounds [11-14]. Biomass polymers, such as chitin, have attracted interest for the substitution of petroleum-based polymers because of their renewability. Chitin takes the form of a nanofiber (NF)-aligned polymer chain by biosynthesis [15]. The utilization of chitin NFs (ChNFs) as gel materials is 
expected to improve the mechanical strength because of their high crystallinity $[16,17]$. In addition, the biocompatibility and biodegradability of chitin are useful characteristics for various applications $[18,19]$. ChNF can be obtained through the disintegration process in water [20]. In particular, the physicochemical properties of $\beta$-ChNF prepared from squid pens significantly change depending on the $\mathrm{pH}$ values immediately before disintegration $[21,22]$ because amino groups on the surface of ChNF are cationized in acidic $\mathrm{pH}$ conditions, electrostatic repulsion and osmotic pressure then interact between ChNFs. As mentioned earlier, while the pore properties of aerogels can be changed by controlling the condition of ice crystal growth and freeze-drying, they can also be changed by the dispersibility of the material. Revealing the relationship between the physicochemical properties of the original $\beta$-ChNF dispersion and the resulting aerogel after lyophilization is important.

In this study, two types of $\beta$-ChNFs differing in dispersibility were prepared in neutral and acidic conditions $(\mathrm{pH}=4)$ and subsequently lyophilized to obtain aerogels. In addition, the influence of the freezing speed on the nano- and micro-structure of the resulting aerogels was investigated. Scanning electron microscopy was performed to characterize the prepared aerogels. A nitrogen adsorption method was used to measure the relative surface area, and compression tests were conducted.

\section{Experimental Section}




\subsection{Materials and preparation of $\beta$-ChNFs}

$\beta$-Chitin was prepared from squid pens (Todarodes pacificus) according to the method reported in our previous paper [23]. In brief, the squid pens were treated with $0.1 \mathrm{~mol} \mathrm{~L}^{-1}$ hydrochloric acid for $16 \mathrm{~h}$ at $15^{\circ} \mathrm{C}$ to remove ash, then twice with $1 \mathrm{~mol} \mathrm{~L}^{-1}$ sodium hydroxide for $2 \mathrm{~h}$ at $90{ }^{\circ} \mathrm{C}$ to remove protein. The purified $\beta$-chitin (95\% $\mathrm{N}$-acetylation) was pulverized into particles of approximately $100 \mu \mathrm{m}$ in diameter using a dry pulverizer (Cyclone Mill, Nippon Steel \& Sumikin Fine Technology Co., Ltd., Osaka, Japan). Hydrochloric acid was purchased from Wako Pure Chemical Industries (Osaka, Japan) and used without further purification. $\beta$-Chitin powders were suspended in distilled water at concentrations of $1 \mathrm{w} / \mathrm{v} \%$. The measured $\mathrm{pH}$ of the prepared slurries was 6.5-7.5. A dilute hydrochloric acid solution was added to the $\beta$-chitin slurries to adjust their acidity before being disintegrated into NFs. The acidity proposed in our previous study, which is the molar ratio of the protons dissociated from the acid to the amino groups in the chitin, is effective for controlling the physicochemical properties of $\beta$-ChNFs [21]. The acidity of the $\beta$-ChNF slurry was set to $0.93 \mathrm{~mol} \mathrm{~mol}^{-1}$ as an acidic condition. Two types of $\beta$-chitin slurries were disintegrated using the Star Burst system (Star Burst Mini, Sugino Machine Co., Ltd., Uozu, Japan) equipped with a ball-collision chamber. The chitin slurries pressurized to approximately $235 \mathrm{MPa}$ were ejected from the nozzle (100- $\mu \mathrm{m}$ aperture) and made to collide with a ceramic ball. The disintegrated samples were cooled at $25{ }^{\circ} \mathrm{C}$ through a heat exchanger, then recovered. The number of collisions with 
the ceramic ball (passes) was set to 10 . The $\beta$-ChNFs prepared in neutral and acidic conditions herein are expressed as $\beta-\mathrm{ChNF}$ [neutral] and $\beta$-ChNF [acid], respectively.

\subsection{Preparation of the aerogels of $\beta$-ChNFs}

Four milliliters of the $\beta$-ChNF [neutral] and [acid] dispersions were loaded into an aluminum-silicone rubber mold (Figs. 1a and b) and sealed with clips. These were immediately frozen by rapidly decreasing the temperature via supercooling to induce the same freezing speed of the two types of $\beta$-ChNF dispersions. In detail, the molds were immersed in 50 vol.\% of water-ethanol refrigerant cooled at $-5{ }^{\circ} \mathrm{C}$ for $1 \mathrm{~h}$ to form the supercooling state (CCA-1110, Tokyo Rikakikai Co., Ltd., Tokyo, Japan). After that, the temperature inside the chiller was set to $-25^{\circ} \mathrm{C}$, and the mold was cooled for an additional $1 \mathrm{~h}$. The temperature inside the mold was directly measured by inserting a thermocouple into the mold. The frozen $\beta$-ChNF dispersions were lyophilized for over 16 h using a freeze-dryer (FDU-2200, Tokyo Rikakikai Co., Ltd.). The obtained aerogel was dried at $70{ }^{\circ} \mathrm{C}$ for $5 \mathrm{~h}$ before further characterization to eliminate moisture. 
(a)

Light blue: silicone rubber
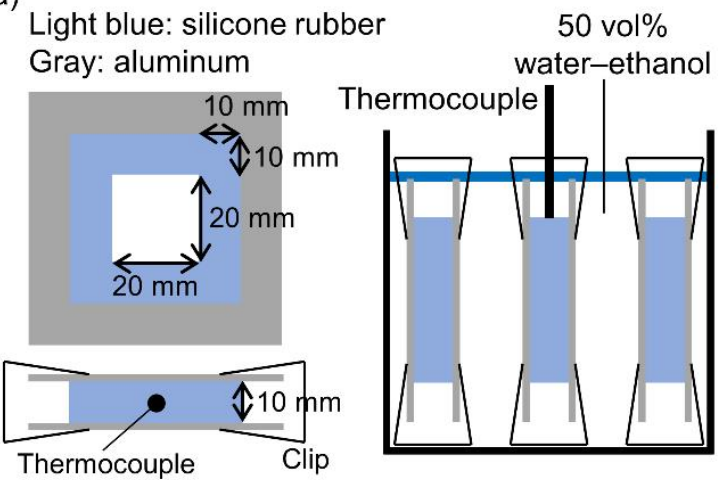

(b)
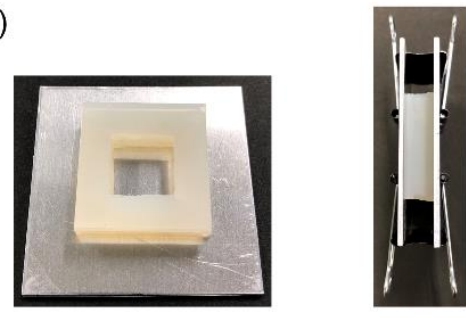

Fig. 1. (a) Schema of the aluminum-silicone rubber mold and chiller. (b) Photographs of the

mold.

\subsection{Scanning electron microscopy observations}

The aerogels were cut to a quarter after cooling with liquid nitrogen. After the specimens were coated with a $5 \mathrm{~nm}$-thick layer of platinum using a sputtering apparatus (JEC-3000FC, JEOL Ltd., Tokyo, Japan), the specimens were observed by a scanning electron microscope (SEM; SU1510, HITACHI Co., Ltd., Tokyo, Japan) operated at a voltage of $3.0 \mathrm{kV}$. Approximately 140 pore surface areas were measured from two different SEM images using the ImageJ software (NIH, Bethesda, USA). The equivalent circle diameter (i.e., pore diameter) was calculated from the measured pore surface area. A histogram of pore diameter was then constructed. In addition, the $\beta$-ChNF dispersions were quickly frozen on an aluminum plane 
cooled with liquid nitrogen, then lyophilized. The pore diameter of this specimen was also measured.

2.4 Relative surface area measurement using the nitrogen adsorption method

The nitrogen adsorption and desorption isotherms of the aerogels were measured with BELSORP-max (MicrotracBEL Corp. Co., Ltd., Osaka, Japan) at $-196{ }^{\circ} \mathrm{C}$ after degassing at $110^{\circ} \mathrm{C}$ for $4 \mathrm{~h}$. The relative surface area was calculated using Brunauer-Emmett-Teller (BET) theory at relative vapor pressures below 0.25 . In addition, the $\beta$-ChNF dispersions were rapidly frozen on an aluminum plane cooled with liquid nitrogen, then lyophilized. The relative surface area of this specimen was also calculated.

\subsection{Compression test}

Compression tests were performed using a TENSILON RTC-1350A (A\&D Company, Ltd., Tokyo, Japan) with a $50 \mathrm{~N}$ load cell. Eighty percent of the aerogel was compressed at a compression rate of $1 \mathrm{~mm} \mathrm{~min}^{-1}$ at $25{ }^{\circ} \mathrm{C}$. The compressive modulus was determined at an initial strain of $4-10 \%$. The energy absorption was calculated as the area below the stressstrain curve until a strain of $70 \%$. The measurements were performed in triplicate. The mean and the standard deviation were also calculated. 


\section{Results and discussion}

Fig. 2 illustrates the temperature profiles inside the mold. $\beta$-ChNF [neutral] and [acid] were maintained in the state of supercooling for $60 \mathrm{~min}$ and subsequently frozen for $2 \mathrm{~min} 52 \mathrm{~s}$ and 3 min $20 \mathrm{~s}$, respectively. The freezing speed was considered almost the same among the two samples, and no difference in the physicochemical properties derived from the freezing speed was observed. Fig. 3 depicts photographs of the $\beta$-ChNF [neutral] and [acid] aerogels. Both aerogels were recovered with the same volume as the mold, that is, shrinkage did not occur.

The stiffness of the $\beta$-ChNF [neutral] aerogel was different between the bottom and center planes in palpation.

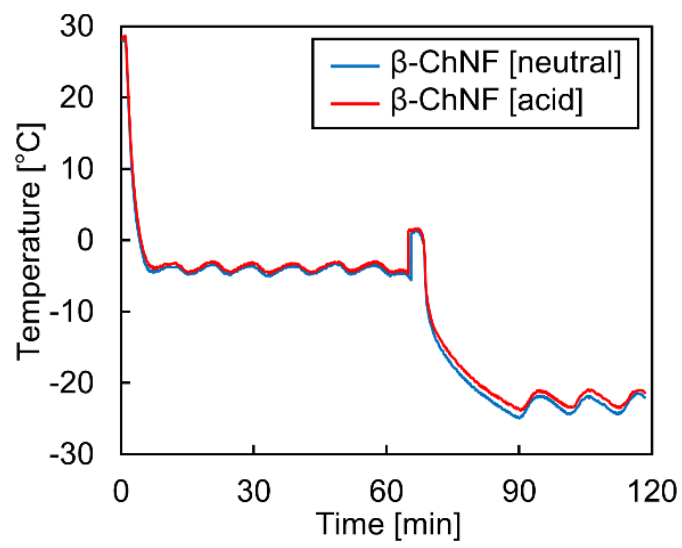

Fig. 2. Temperature profiles inside the mold for freezing $\beta$-ChNF [neutral] and [acid] dispersions.

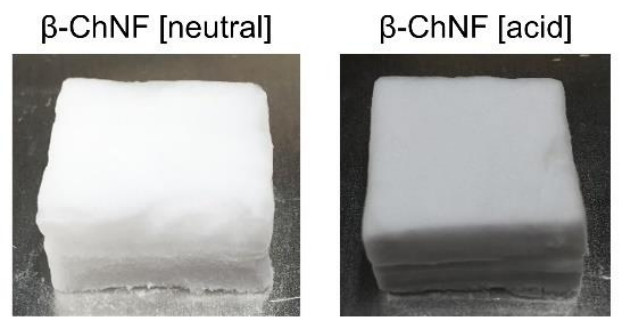

Fig. 3. Photographs of the aerogels obtained after lyophilization. 
Fig. 4a shows the SEM images and pore diameter distributions of the aerogels. The $\beta$-ChNF [neutral] aerogel consisted of a stacking structure of aggregation sheets with macro pores. This structure was the result of NFs aggregation among the ice crystals during the water solidification. In contrast, the $\beta$-ChNF [acid] aerogels possessed smaller sheets, and the roughness of the surface increased. The average pore diameter also decreased in half. The decrease in the pore diameter was derived from the surface charge of the ChNF. The amino groups existing on the $\beta$-ChNFs were cationized in acidic conditions, and the electrostatic repulsion and osmotic pressure interacted between the $\beta$-ChNFs. Indeed, there is a previous report that the widths of $\beta$-ChNFs prepared in neutral and acidic conditions were 27.3 and 12.1 $\mathrm{nm}$, respectively; that is, repulsions between $\beta$-ChNFs resulted in thin NF widths [21]. These repulsions exerted influence during ice crystal growth; as a result, microsized sheets were formed. It has been reported that the pore diameter suited to the cell culture was $50-150 \mu \mathrm{m}$ $[24,25]$. The $\beta$-ChNF [neutral] aerogel was likely more suitable than the $\beta$-ChNF [acid] aerogel as a scaffold in terms of only the pore diameter. In addition, the freezing speed significantly influenced the pore diameter. From the result of quick-freezing with liquid nitrogen, the pore diameter decreased by approximately one sixth or one seventh. This result indicated that the ice crystal did not sufficiently grow because the freezing speed was extremely quick. In addition, the influence of the repulsion by the positive charge can be observed with quick- 
freeze, as shown in Fig. 4b. In the $\beta$-ChNF [acid] aerogel with quick-freeze, the NF structure remained in the macro pores without the aggregation and existed between microsized sheets, similar to cobwebs. In contrast, the enlarged view of the $\beta$-ChNF [neutral] showed many microsized sheets formed by the aggregation of NFs, although a few NFs were observed.

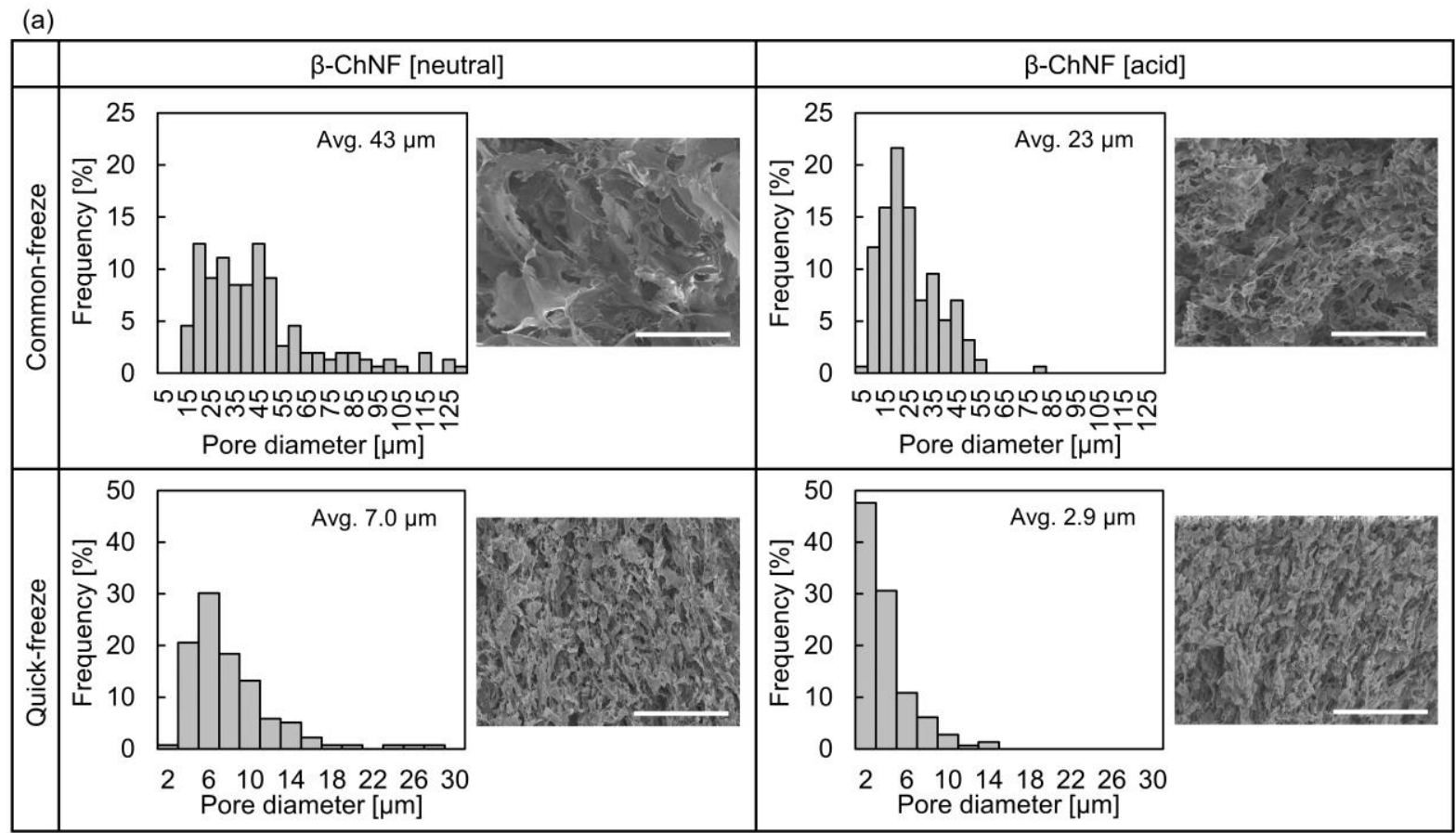

(b)

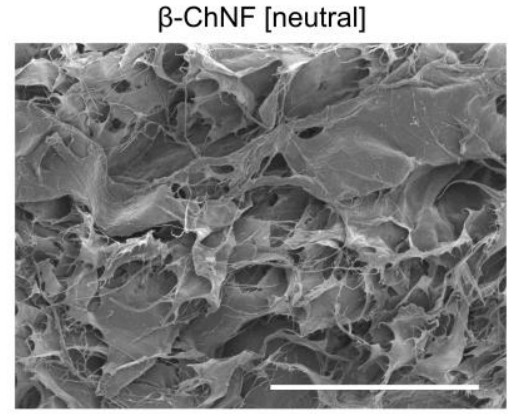

$\beta-C h N F[$ acid]

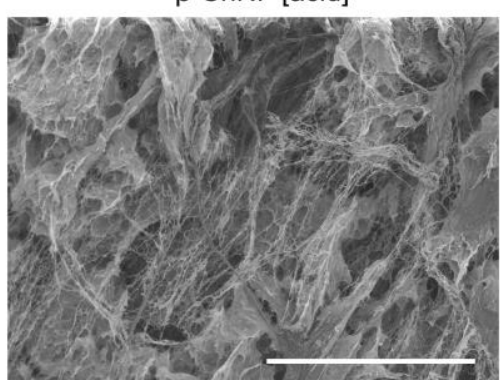

Fig. 4. (a) SEM images of the aerogels and histograms of the pore diameter. The scale bars in the SEM images indicate $200 \mu \mathrm{m}$. (b) Enlarged view of the quick-freeze aerogels. The scale bars in the SEM images indicate $50 \mu \mathrm{m}$. 
Fig. 5 shows the nitrogen adsorption and desorption isotherms of the aerogels and the relative surface area calculated from the BET equation. These isotherms represent type-II in the IUPAC classification, namely, there are more macro pores (over $50 \mathrm{~nm})$ than meso pores $(2-50 \mathrm{~nm})$ [26]. This result corresponded to the SEM observation. The relative surface area of the $\beta$-ChNF [acid] aerogel was more than two times greater than that of the $\beta$-ChNF [neutral] aerogel. The relative surface area of the respective aerogel prepared by the quick-freeze method was more than three times greater than that of respective former aerogels. The aerogels with a larger pore diameter showed a smaller relative surface area. Interestingly, the relative surface area of the original $\beta$-chitin powder and the $\beta$-ChNF [neutral] aerogel was almost the same. After the $\beta$ chitin powder was disintegrated into NFs, the NFs aggregated again until reaching the same level of powder by ice crystal growth. In other words, the morphological transition from the powder to the shape of the mold took place. Although the ChNF dispersions were molded into the rectangular shape, those could be transformed into arbitrary shapes depending on the mold shape. 
(a)

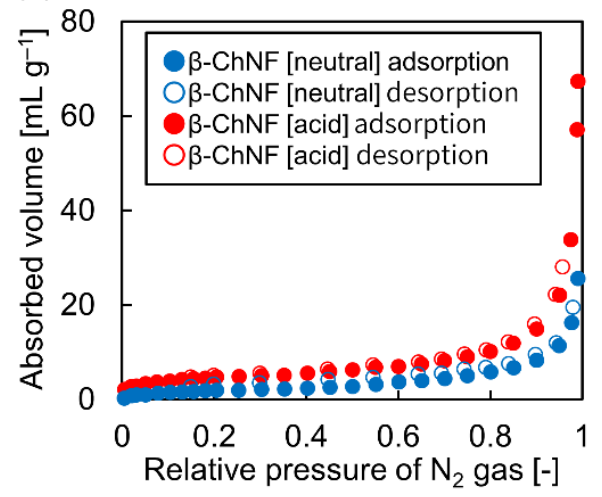

(b)

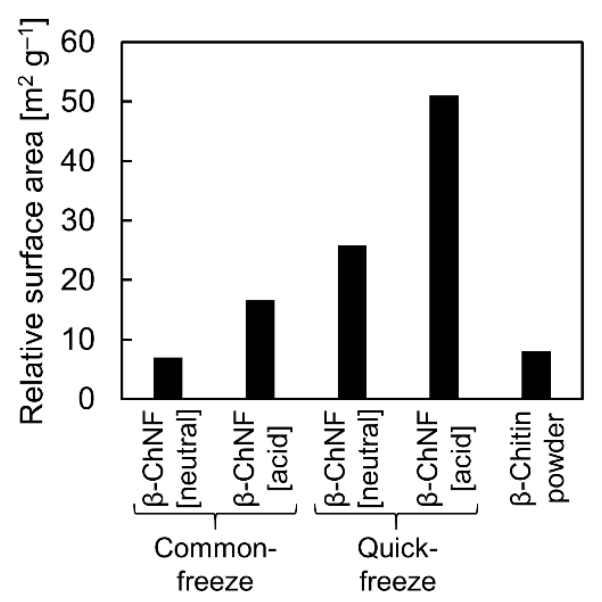

Fig. 5. (a) Nitrogen adsorption and desorption isotherms and (b) relative surface area of the aerogels.

Fig. 6 shows the stress-strain curves of the aerogels. Table 1 lists the mechanical properties of the aerogels. Both bulk densities were almost identical and had values as close as $1 \mathrm{wt} . \%$ of the original $\beta$-ChNF dispersion concentration because shrinkage did not occur during the aerogel preparation, as described earlier. However, the compressive modulus and the energy absorption of the $\beta$-ChNF [acid] aerogels were 30 and three times greater than those of the $\beta$ ChNF [neutral] aerogels, respectively, probably because of the aerogel uniformity. As described earlier, the strength of the $\beta$-ChNF [neutral] aerogel was different between the bottom 
and central planes, and the bottom plane was easily compressed. The bottom plane of the mold was made of aluminum, and the side plane was made of silicone rubber; therefore, the freeze proceeded from both bottom planes according to the high thermal conductivity of aluminum. The nonuniformity of NFs by ice crystal growth proceeded to the central plane, resulting in dense aggregation at the central plane. Thus, the stress-strain curve of the $\beta$-ChNF [neutral] aerogel cannot be distinguished between the elastic deformation region and the plastic deformation region, and the mechanical strength showed small values. In contrast, the $\beta$-ChNF [acid] aerogel possessed a uniform structure because of the electrostatic repulsion. As a result, the elastic deformation region was clearly observed, and the compressive modulus was high. It has been reported that chitin/silk composites with a compressive modulus of $60 \mathrm{kPa}$ were used to cell-culture the fibroblast of a rat in vitro [27]. The $\beta$-ChNF [acid] aerogels are likely suitable for scaffolding in terms of mechanical strength.

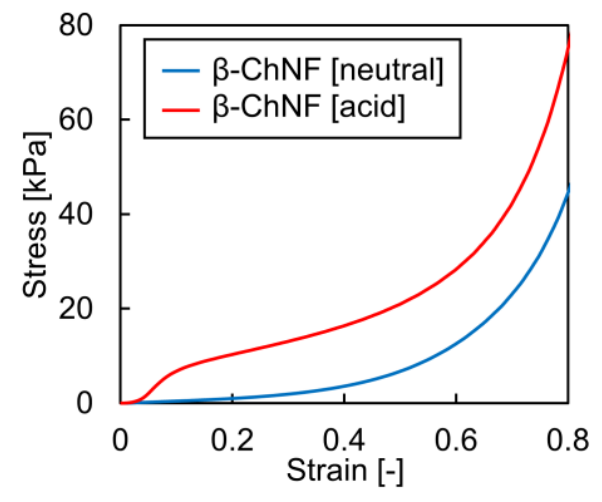

Fig. 6. Stress-strain curves for the aerogels.

Table 1. Mechanical properties of the aerogels. 


\begin{tabular}{cccc}
\hline Sample & $\begin{array}{c}\text { Bulk density } \\
{\left[\mathrm{mg} \mathrm{cm}^{-3}\right]}\end{array}$ & $\begin{array}{c}\text { Compressive modulus } \\
{[\mathrm{kPa}]}\end{array}$ & $\begin{array}{c}\text { Energy absorption } \\
{\left[\mathrm{kJ} \mathrm{m}^{-3}\right]}\end{array}$ \\
\hline$\beta$-ChNF [neutral] & $9.73 \pm 0.30$ & $3.89 \pm 1.81$ & $3.42 \pm 0.30$ \\
$\beta$-ChNF [acid] & $9.24 \pm 0.31$ & $109 \pm 8$ & $11.2 \pm 0.6$ \\
\hline
\end{tabular}

Means of three experiments \pm standard deviation.

\section{Conclusions}

$\beta$-Chitin aerogel was produced from $\beta$-ChNF dispersions via lyophilization. Two types of $\beta$ -

ChNF dispersions were prepared in neutral and acidic conditions and had different surface

charges. The $\beta$-ChNFs [neutral] were moved to the gaps between the ice crystals during solidification and formed microsized sheets. The average pore diameter was $43 \mu \mathrm{m}$, which is suitable for cell cultures in terms of vascularization and cellular viability. The surface area of the $\beta$-ChNFs [neutral] was almost the same as that of the original $\beta$-chitin powder because of the morphology transformation from the powder to the mold shape. However, the $\beta$-ChNFs [neutral] were nonuniformly aggregated from the bottom plane toward the central plane. In contrast, the $\beta$-ChNF [acid] aerogels possessed high relative surface area and high mechanical strength compared to the $\beta$-ChNF [neutral] aerogels. The amino groups on the $\beta$ ChNFs were cationized in acidic conditions, and there were repulsions between the NFs. The repulsion was highly effective during the lyophilization process, and the NF structure remained in the macro pores. As a result, the $\beta$-ChNF [acid] aerogels consisted of minute micro-sheets and a uniform structure, resulting in a significant increase in the mechanical 
strength. In the future, chemical and thermal stability as well as biodegradability of the $\beta$ ChNF aerogels will be studied. For example, the lyophilized materials in this study were made to shrink in water at ordinary temperatures. In addition, the degree of $N$-acetylation and the freezing speed and direction should be studied to prepare aerogels with higher strength and ideal pore structure. 


\section{Acknowledgments}

Funding: This work was supported by JSPS KAKENHI [grant number 17H04893]. We thank

Dr. Nobuhide Takahashi, Dr. Hiroshi Fukunaga, Dr. Iori Shimada, Dr. Kazuhide Totani, Dr.

Yoshihiro Nomura, and Mr. Kazuhiko Yamashita for their substantial intellectual contributions to the conception of the project.

\section{Conflict of Interest}

The authors declare that they have no conflict of interest. 


\section{References}

[1] L. Heath, W. Thielemans, Cellulose nanowhisker aerogels, Green Chem. 12 (2010) 1448-1453. doi:10.1039/c0gc00035c.

[2] B. Ding, J. Cai, J. Huang, L. Zhang, Y. Chen, X. Shi, Y. Du, S. Kuga, Facile preparation of robust and biocompatible chitin aerogels, J. Mater. Chem. 22 (2012) 5801-5809. doi:10.1039/c2jm16032c.

[3] A.E. Donius, A. Liu, L.A. Berglund, U.G.K. Wegst, Superior mechanical performance of highly porous, anisotropic nanocellulose-montmorillonite aerogels prepared by freeze casting, J. Mech. Behav. Biomed. Mater. 37 (2014) 88-99. doi:10.1016/j.jmbbm.2014.05.012.

[4] Y. Kobayashi, T. Saito, A. Isogai, Aerogels with 3D ordered nanofiber skeletons of liquid-crystalline nanocellulose derivatives as tough and transparent insulators, Angew. Chem. Int. Ed. Engl. 53 (2014) 10394-10397. doi:10.1002/anie.201405123.

[5] L. Lewis, M. Derakhshandeh, S.G. Hatzikiriakos, W.Y. Hamad, M.J. MacLachlan, Hydrothermal gelation of aqueous cellulose nanocrystal suspensions, Biomacromolecules 17 (2016) 2747-2754. doi:10.1021/acs.biomac.6b00906.

[6] B. Duan, H. Gao, M. He, L. Zhang, Hydrophobic modification on surface of chitin sponges for highly effective separation of oil, ACS Appl. Mater. Interfaces 6 (2014) 19933-19942. doi:10.1021/am505414y. 
[7] F. Zhang, H. Ren, G. Tong, Y. Deng, Ultra-lightweight poly (sodium acrylate)

modified TEMPO-oxidized cellulose nanofibril aerogel spheres and their superabsorbent properties, Cellulose 23 (2016) 3665-3676. doi:10.1007/s10570-0161041-8.

[8] M. Li, C. Zhang, S. Lu, Z. Wu, H. Yan, Study on porous silk fibroin materials: 3. Influence of repeated freeze-thawing on the structure and properties of porous silk fibroin materials, Polym. Adv. Technol. 13 (2002) 605-610. doi:10.1002/pat.159.

[9] A.J. Svagan, L.A. Berglund, P. Jensen, Cellulose nanocomposite biopolymer foamhierarchical structure effects on energy absorption, ACS Appl. Mater. Interfaces 3 (2011) 1411-1417. doi:10.1021/am200183u.

[10] Z.-Z. Pan, H. Nishihara, S. Iwamura, T. Sekiguchi, A. Sato, A. Isogai, F. Kang, T. Kyotani, Q.-H. Yang, Cellulose nanofiber as a distinct structure-directing agent for xylem-like microhoneycomb monoliths by unidirectional freeze-drying, ACS Nano. 10 (2016) 10689-10697. doi:10.1021/acsnano.6b05808.

[11] T.M. Tillotson, L.W. Hrubesh, Transparent ultralow-density silica aerogels prepared by a two-step sol-gel process, J. Non-Cryst. Solids. 145 (1992) 44-50. doi:10.1016/S0022-3093(05)80427-2.

[12] J. Zhang, G. Chen, Q. Zhang, F. Kang, B. You, Self-assembly synthesis of N-doped carbon aerogels for supercapacitor and electrocatalytic oxygen reduction, ACS Appl. 
Mater. Interfaces 7 (2015) 12760-12766. doi:10.1021/acsami.5b01660.

[13] G. Hayase, K. Nonomura, G. Hasegawa, K. Kanamori, K. Nakanishi, Ultralowdensity, transparent, superamphiphobic boehmite nanofiber aerogels and their alumina derivatives, Chem. Mater. 27 (2015) 3-5. doi:10.1021/cm503993n.

[14] Y. Tamada, New process to form a silk fibroin porous 3-D structure, Biomacromolecules 6 (2005) 3100-3106. doi:10.1021/bm050431f.

[15] D. Raabe, C. Sachs, P. Romano, The crustacean exoskeleton as an example of a structurally and mechanically graded biological nanocomposite material, Acta Mater. 53 (2005) 4281-4292. doi:10.1016/j.actamat.2005.05.027.

[16] C. Chen, D. Li, Q. Hu, R. Wang, Properties of polymethyl methacrylate-based nanocomposites: Reinforced with ultra-long chitin nanofiber extracted from crab shells, Mater. Des. 56 (2014) 1049-1056. doi:10.1016/j.matdes.2013.11.057.

[17] S. Suenaga, M. Osada, Parameters of hydrothermal gelation of chitin nanofibers determined using a severity factor, Cellulose, 25 (2018) 6873-6885. doi:10.1007/s10570-018-2053-3.

[18] R. Jayakumar, M. Prabaharan, P.T.S. Kumar, S.V. Nair, H. Tamura, Biomaterials based on chitin and chitosan in wound dressing applications, Biotechnol. Adv. 29 (2011) 322-337. doi:10.1016/j.biotechadv.2011.01.005.

[19] R. Izumi, S. Komada, K. Ochi, L. Karasawa, T. Osaki, Y. Murahata, T. Tsuka, T. 
Imagawa, N. Itoh, Y. Okamoto, H. Izawa, M. Morimoto, H. Saimoto, K. Azuma, S.

Ifuku, Favorable effects of superficially deacetylated chitin nanofibrils on the wound healing process, Carbohydr. Polym. 123 (2015) 461-467.

doi:10.1016/j.carbpol.2015.02.005.

[20] S. Ifuku, M. Nogi, K. Abe, M. Yoshioka, M. Morimoto, H. Saimoto, H. Yano, Preparation of chitin nanofibers with a uniform width as $\alpha$-chitin from crab shells, Biomacromolecules 10 (2009) 1584-1588. doi:10.1021/bm900163d.

[21] S. Suenaga, K. Totani, Y. Nomura, K. Yamashita, I. Shimada, H. Fukunaga, N. Takahashi, M. Osada, Effect of acidity on the physicochemical properties of $\alpha$ - and $\beta$ chitin nanofibers, Int. J. Biol. Macromol. 102 (2017) 358-366. doi:10.1016/j.ijbiomac.2017.04.011.

[22] S. Suenaga, M. Osada, Systematic dynamic viscoelasticity measurements for chitin nanofibers prepared with various concentrations, disintegration times, acidities, and crystalline structures, Int. J. Biol. Macromol. 115 (2018) 431-437. doi:10.1016/j.ijbiomac.2018.04.082.

[23] S. Suenaga, N. Nikaido, K. Totani, K. Kawasaki, Y. Ito, K. Yamashita, M. Osada, Effect of purification method of $\beta$-chitin from squid pen on the properties of $\beta$-chitin nanofibers, Int. J. Biol. Macromol. 91 (2016) 987-993. doi:10.1016/j.ijbiomac.2016.06.060. 
[24] B.S. Chang, C.K. Lee, K.S. Hong, H.J. Youn, H.S. Ryu, S.S. Chung, K.W. Park, Osteoconduction at porous hydroxyapatite with various pore configurations, Biomaterials 21 (2000) 1291-1298. doi:10.1016/S0142-9612(00)00030-2.

[25] I. Sopyan, M. Mel, S. Ramesh, K.A. Khalid, Porous hydroxyapatite for artificial bone applications, Sci. Technol. Adv. Mater. 8 (2007) 116-123. doi:10.1016/j.stam.2006.11.017.

[26] K.S.W. Sing, D.H. Everett, R.A.W. Haul, L. Moscou, R.A. Pierotti, J. Rouquérol, T. Siemieniewska, Reporting physisorption data for gas/solid systems with special reference to the determination of surface area and porosity, Pure Appl. Chem. 57 (1985) 603-619. doi:10.1351/pac198557040603.

[27] P. Wongpanit, N. Sanchavanakit, P. Pavasant, T. Bunaprasert, Y. Tabata, R.

Rujiravanit, Preparation and characterization of chitin whisker-reinforced silk fibroin nanocomposite sponges, Eur. Polym. J. 43 (2007) 4123-4135. doi:10.1016/j.eurpolymj.2007.07.004. 


\section{Tables}

Table 1. Mechanical properties of the aerogels.

\begin{tabular}{cccc}
\hline Sample & $\begin{array}{c}\text { Bulk density } \\
{\left[\mathrm{mg} \mathrm{cm}^{-3}\right]}\end{array}$ & $\begin{array}{c}\text { Compressive modulus } \\
{[\mathrm{kPa}]}\end{array}$ & $\begin{array}{c}\text { Energy absorption } \\
{\left[\mathrm{kJ} \mathrm{m}^{-3}\right]}\end{array}$ \\
\hline$\beta$-ChNF [neutral] & $9.73 \pm 0.30$ & $3.89 \pm 1.81$ & $3.42 \pm 0.30$ \\
$\beta$-ChNF [acid] & $9.24 \pm 0.31$ & $109 \pm 8$ & $11.2 \pm 0.6$ \\
\hline
\end{tabular}

Means of three experiments \pm standard deviation. 


\section{Figure Captions}

Fig. 1. (a) Schema of the aluminum-silicone rubber mold and chiller. (b) Photographs of the mold.

Fig. 2. Temperature profiles inside the mold for freezing $\beta$-ChNF [neutral] and [acid] dispersions.

Fig. 3. Photographs of the aerogels obtained after lyophilization.

Fig. 4. (a) SEM images of the aerogels and histograms of the pore diameter. The scale bars in the SEM images indicate $200 \mu \mathrm{m}$. (b) Enlarged view of the quick-freeze aerogels. The scale bars in the SEM images indicate $50 \mu \mathrm{m}$.

Fig. 5. (a) Nitrogen adsorption and desorption isotherms and (b) relative surface area of the aerogels.

Fig. 6. Stress-strain curves for the aerogels.

Figs. 1-6 are provided as PowerPoint files 